5.

\title{
Ein Fall von paralytischer Geisteskrankheit mit Degeneration der Hirurinde, Sklerom des kleinen Gelirns und partieller obliteration des Circulus Willisii.
}

\author{
Von Dr. Franz Meschede, \\ zweitem Arzte der Prowinzial-Kranken-Anstalten zu Schwetz.
}

Die Geschichte dieses ebensowohl durch die Mannigfaltigkeit und Schwere der ätiologischen Momente als der pathologischen Befunde ausgezeichneten Falles von paralytiseher Geisteskrankheit ist kurz folgende:

Auf dem Boden einer hereditären und acquirirten Disposition batte sich die Krankheit aus Veranlassung eines Concurses, der üher das Vermögen des Kranken $X$, damals Inhaber eines bedeutenden Weingeschäftes, hereingebrochen war, zunächst unter dem Bilde der Melancholie entwickelt. Nach den vorliegenden ärztlichen Zeugnissen soll der Vater eine "fixe lỏee" gehabt baben, die Hatter aber "vollstăndig verrïckt" gewesen sein. Ueber den Kranken selbst wird berichtet, dass er Ausschweifungen in Ven. et Baceho in unmässigem Grade ergeben gewesen sei und dadurch, sowie durch mehrere syphilitische Ansteckungen und durch Onanie seine Constitution in hohem Grade und „bis zur vollständigen Impotenz “ geschwächt habe.

Im Laufe des Jahres 1861, kurze Zeit nach dem Falle seines Hauses, bemerkte man an ihm eine grosse Niedergeschlagenheit und Zerstreutheit, ein ängstliches und unstätes Wesen, eine zwecklose Geschäftigkeit und eine allmählich zunehmende Geistesschwäche. Zugleich stellte sich fast andauernde Schlaflosigkeit ein. Ganze Nächte hindurch stöberte und kramte er in seinen Concurs-Acten, ohne ein Ende finden zu können. Dieser Zustand innerer Beunruhigung steigerte sjch allmählich, erreichte im October genannten Jabres seinen Höhepunkt und schlug dann in die entgegengesetzte Gefühlslage um: an stelle der Beengung und Depression trat Expansion und Exaltation des Selbstgefühls und gleichzeitig machte sich eine excessive Geschlechts-Aufregung bemerklich. Doch wurde die heitere Stimmung noch intercurrent durch kurze Perioden tiefer Verstimmung sowie durch Zornwuth-Paroxysmen unterbrochen. Nach dern vergeblichen Gebrauche einer Kaltwasser-Kur machte die geistige Zerrüttung in folgenden Jahre (1862) grössere Fortschritte: Es traten ausgeprägter Grössenwahn, insbesondere Ideen grossen Reichthums und gleichzeitig anch Lähmung-Erscheinungen za Tage and im Juni 1862 stellten sich auch drei paralytische Anfälle (Epilepsie-ähnliche mit bewusstlosem Niedersinken verbundene klonische Krämpfe der Glieder von etw: 15 Minuten Daver) ein.

Bei der demnächst im Juli 1862 erfolgten Aufnahme in die biesige Anstal bot Pat. bereits das Bild vorgeschrittenen paralytischen Blödsinns dar. I dem ganzen äusseren Habitus des etwa mittelgrossen Kranken spiegelte sich "himm lisches Behagen" wieder, unbedingte Daseinswonne und Daseinsbarmonie. Dt durchsichtige blonde Téint des mit alten Pockennarben bedeckten Gesichtes erschie durch eine mässige Turgescenz geröthet, die Physiognomie athmete eín freundlich 
Wohlwollen, die etwas näselnde, affectroll gedehnte Sprache verliündete ebenfalls das Vorberrschen einer freudigen Seelenstimmung, die unstäte Hast seiner Bewegungen eine abnorme Erregung der motorischen Sphäre und eine Art vornebmer Herablașsung die Gehobenheit des Selbstgefühls. Er schwelgte in Grössen-Ideen, in Ueppigkeit und Comfort, rülunte seine bedeutenden geistigen Fähigkeiten, seine grossen Erfolge als Musik-Virtuose, erzäblte u. A., dass er zur Cur seines Armes "immel 90 Fontanellen auf einmal belommen babe u.s.w. Er hält sich nicht als Kranker, sondern nur "vergnügungshalber" in der Anstalt auf, findet den Aufenthalt darin übrigens "entzückend". - Die linke Pupille war stark erweitert, die Sehkraft des rechten Auges gesebwächt, das linke Ellenbogengelenk ankylotisch, der Appetit, Schlaf und die Verdaung gut, die Haut turgescirt und warm.

Am 14. October 1862 erlitt Pat. auf dem Spaziergange plötzlich einen paraIytischen Anfall; die Sprache, Zunge und die linken Extremitäten erschienen vollständig paralysirt, die rechten Extremitäten paretisch, beíde Pupillen dilatirt; der Puls schlug langsam und schwach, das Secsorium war etwas benommen. In der Nacht und am folgenden Tage stellte sich Kopfschmerz ein, das Gesicht erschien deturgescirt, collabirt, die Körpertemperatur verringert, die Unfähigkeit die Zunge hervorzustrecken and die linken Extremitäten zu gebrauchen dauerte fort. In der Nacht rom 15ten zum 16ten: Zuckungen des linken Armes und linken Beines, mangelhaft articulirte und unverständliche Sprache; am 16ten konnte Pat. die Zunge wieder hervorstrecken und am 18 ten und 19 ten die bisher gelähmten Extremitäten wieder einigermaassen gebrauchen.

Am 20sten war Pat. bereits soweit gekrältigt, dass er das Bett verlasseñ und wieder verständlich, wenngleich mit einiger Schwierigkeit sprechen konnte. Bemerkenswerth war die nun eintretende hohe Steigerung des Selbstgefühls und Exacerbation des Grössenwahns. Mit einer bis dahin bei ihm noch nicht beobachteten Emphase sprach der Kranke von seinen vielen und schönen Frauen, seinen herrlichen Beinkleidern u. s. w., fühlte eine grössere Wärme und ein himmlisches Wonnegefühl seinen Körper durchströmen, insbesondere auch starken Geschlechtstrieb, stieg nackt aus dem Bette und verweilte in kriechenden und anderen sonderbaren Attitüden, zuweilen onanirend und wie in einem trunkenen und geistesabwesenden Zustande, ganz in Wollust versunken, so dass er unter ununterbrochener Aufsicht gehaiten werden musste.

Am 23. October leichter paralytischer Anfall, besonders charakterisirt durch Zuckungen des recbten Armes, Benommenheit des Sensoriums, Verlangsamung der geistigen Bewegung, Verlangsamung des Pulses (68), normale Körpertemperatur. Am 30. October ercenter paralytiscber Anfall: allgemeine Hinfälligkeit, Benommenheit des Sensoriums, vollständige Sprachlähmung, allgemeiner Collapsus.

Trotz vorübergehender Besserung, welche sich nach Anwendung localer Blutentziehungen unḋ Derivantien einstellte, erschienen die Geistes- und Körperkräfte doch nach diesem Anfalle in einem viel bedeutenderen Grade als nach den früheren Anfällen gesunken. Pat. blieb von diesem Zeitpunkte an dauernd unfähig, das Bett zu verlassen, lag in einer Art Halbschlummer, halbträumend und blödsinnig lächelnd, immermehr das Bild geistiger Nullität gewäbrend. 
Seit Anfang Januar 1863 erschien die rechte Pupille dilatirt. Am 3. Februar paralytischer Anfall von etwa $\frac{1}{2}$ Minute Dater: Zuckungen der Extremitäten und der Hals- und Kopfmuskeln, besonders linkerseits, auch in den folgenden Tagen einige Male wiederkehrend. Hierauf wäbrend 5 Tage gänaliche Unfähigkeit zu sprechen, and Lähmung der willkürichen Bewegung der Extremitäten. Am 27. März convulsivische Zuckungen beider Arme.

Am 5. April intermittirende Erweiterung der linken Pupille, nämlich: von 6 Uhr bis $\frac{1}{2} 11$ Uhr Vormittags, von $3 \frac{1}{2}$ Uhr Nachmittags bis zum anderen Tage 7 Uhr Morgens. Gänzliche Unbesinnlicbkeit und Sprachlosigkeit, Mangel jeglichen Verständuisses, complete allgemeine Lähmung. Nach einigen Tagen Nachlass der Lähmungs-Erscheinungen und Rückkehr der Fähigkeit zu sprechen.

Von Mitte April 1863 bis Mitte Mai sprach Pat. nicht ein einziges Wort und gab überbaupt keinerlei Zeichen eines Verstandnisses.

Am 13. Mai Abends steliten sich leichte Zuckungen der linken Gesichtshälfte ein, die sich am 14 ten Morgens und Nachmittags, in der folgenden Nacht und am 16.-17. Mai wiederholten. Zugleich kehrte die Sprache zurück und Pat. erwachte zu neuer Redseligkeit.

Am 13. Juni Abends starke Zuckunged der linken Gesichtshälfte, dann Zuckungen der recbten Seite und Erweiterung der rechten Papille.

Im Verlaufe des Juni und Juli traten die Erscheinungen organischer Dissolution in den Vordergrund: 0thaematom der rechten Seite, allmählich bis zur Grösse einer Kartoffel wachsend, Furunculosis, Erythem und Oedem der Scrotalbaut, Excoriationen an der Glans p, Oedema praeputii, oberlächlicher Druckbrand, ferner tuberculöse Infiltration der Lungen, rapider Verfall der Körper- und Geisteskräfte, Abmagerung; gegen Ende Juni Schlundlähmung. Der Tod erfolgte am 9. August 1863 Nachmittags 6 Thr.

Die 23 Stunden nach dem Tode rorgenommene.Section, welche sich auf die Eröt̊nung der Kopfhöhle beschränken musste, ergáb folgenden Befund: Oberfächjicher Sacral-Decubitus, mässiges Oedema pedum, Ankylose des linken Ellenbogengelenks, daselbst eine guldengrosse hrandige Partie; an der Glans Spuren von Narben. In Unterbantgewebe $z$ wischender zweiten und dritten hippe, nabe dem Brustbein, befindet sich eine haselnussgrosse knorpelige Geschwulst, welche unter dem Milsoskope deutliche Knorpelzellen zeigt und nirgends mit dem Rippenknorpel zusammenhängt. Othae. matom rechts.

Schädel ziemlich symmetrisch, schwer, von geringem Blutgehalte; grösste Längsdürchmesser $7 \frac{1}{4}$ Zaill, grösster Breitendurchmesser $\tilde{5}_{4}^{3}$ Zoll. Innere Hyperostos des Schädeldachs, besonders des Stirnbeins, welches an einzelnen. Stellen 6 Linie: dick ist. In Folge der Verdickung der inneren Knochentafel erscheinen die Jug und Impressiones flach, die Nahte verstrichen. An der inneren Tafel des Stirr beins mehrere kleine scharfspitzige 0steophyten. Stirnhöhlen sehr geräumig. Dura m. längs der Pfeilnabt mit dem Schädel verwachsen, auf der Convexität d grossen Gehirns etwas faltig. Innere Oberfiäche glatt; in den beiden vorderen ur. bauptsächlich auch in den Temporalgruben Spuren rost farbener Pigmentauflagerun Eine vollsiändig ausgeprägte, ehenfalls rostfarben pigmentirte dünne Pseudomembr: 
von offenbar älterem Datum befindet sich an der inneren Oberfläche desjenigen Theils der Dura mater, welcher der Convexität des binteren Lappens der rechten Grosshirnhemisphäre entspricht. Sämmtliche Sinus leer, nur in Sin. transvers. etwas Serum und Blut. Auf dem Cliv. Blumenbachii befindet sich in der Mittellinie, $\frac{1}{2}$ Zoll von der Sella turc. entlernt und bedeckt ron der Dura m., ein elwa erbsengrosses 0 steophyt. Die diesem letzteren entsprechende Partie der Arachnoidea ist beträchtlich verdickt und fester als gewöhnlich am Pons adhärent. An derselben Stelle ist auch die Art. basilaris beträchtlich atheromatös verdickt; das Lumen derselben ebendort zugleich verengt. Besonders ist dieses an der Bifurcation der Fall, in deren Winkel das Osteophyt hineinragt. Die Arteria e communic. post. des Circ. Will. erscbeinen als zwei sehr gespante Fäden von etwa Schweinsborstendicke und vollständig blatleer. Die rechte ist $7 \frac{3}{4}$ Zoll lang und vollständig obliterirt, die linke $10 \mathrm{Zoll}$ lang und noch mit einem sehr engen, etwa der Dicke eines feinen Haares entsprechenden Lumen versehen.

Geringer Blutgehalt der weichen Häute, beträchtliches Oedem derselben und der subarachnoidealen Ränme, hauptsächlich in den Sulcis der convexen Oberfläche des grossen Gehirns. Arachnoidea in ihrer ganzen Ausdehnung getrübt und vielfach auch verdickt, an ejnzelnen Stellen der Stirn- und der Temporal-Lappen, besonders an der unteren Flăche und an den spitzen derselben, sowie längs der Sichelränder mitsammt der Pia m. mit der Gehirnrinde verklebt resp. verwachsen. Die Substanz des Gehirns ödematös und anämisch; die Corticalsubsfanz einzelner Gyri des grossen Gehirns atrophisch; die oberste Schicht der Corticalsubstanz grösstentheils von ziemlich normalem Aussehen; die innerste Schicht dagegen in ausgedehnten Bezirken besonders auf der Convexităt anämisch, blass und fahlgelb, stellenweise auch ein missfarbig-graues Colorit zeigend, von derbem Gefäge. Beide Ventrikel beträchtlich erweitert, mit klarer Flüssigkeit angefüllt ; Ependym verdickt, fein granulirt, über dem Corp. striat. mit weissen Flecken versehen. Medullarsubstanz von ziemlich fester Consistenz.

An der oberen Fläche des kleínen Gehirns befindet sich rechts vom Oberwurm and diesem parallel verlanfend eine $\frac{3}{4}$. Zoll lange verlärtete und eingesunkene Partie; dieselbe besteht aus einer Anzabl verschrumpfter und knorpelhart anzufühlender Kleinhirn-Windungen, mit deren Oberfäche die weichen Häute fest verwachsen sind. Beim Durchsehneiden zeigt sich, dass die Verhärtung etwa 1 Zoll weit in die Tiefe reicht und aus drei verschiedenen Schichten besteht, von denen die oberste, etwa 2 Linien dicke, durch die zusammengeschrumpfte und verbärtete Rindensubstanz gebildet wird; die mittlere, etwa 1 Linie mächtig, eine weissliche, derbe, bindegewebige Schwiele darstellt, die innerste dagegen von weicherer Consistenz und fabl-ockergelblicher bis leicht rostgelber Färbung, anscheinend aus veränderter Medullarsubstanz besteht. Diese letztere bildet gleichsam den Kern, weleber von der ebengenannten mitteren Schicht wie von eiver Kapsel umgeben ist und in welchen von letzterer strahlige Faserzüge eingreifen. Auch die übrigen Theile des Cerebellum haben eine rermebrte Consistenz, ebenso das verlängerte Mark. Die Tela chorioid. infer. ist etwas verdickt.

Die mikroskopische Untersuchung, zeigte vorgeschrittene fettige Degeneration der Nervenzellen und zum Theil Zerfall derselben zu bald mehr bald weniger umschriebenen oder diffus zerstreuten Körnermassen. Auch die $\mathrm{Neu}$ roglia zeigte sich in diesem Falle deutlich verändert, insofern sie dunkler und im Allgemeinen auch grobkörniger erschien, viele glänzende und das Licht stark brechende verhältnissmässig grosse Fettkörnchen und in weiter Verbreitung zerstreut Pigmentmoleküle, kleine Partikel von verschiedenen Formen erkennen liess, 
iusbesondere Schollen und unregelmässig gestaltete Körner, deren Färbung sich in den Nüançen zwischen Blassgelbroth und Schwarzbraun bewegte, die aber am häufigsten rostfarben erschienen.

Aebnliche Massen' fanden sich in gleichfalls disseminirter Verbreitung zwischen normalen Elementen auch in der blass-ockerfarbenen Partie, welche den von einer bindegewebigen schwieligen Masse umgebenen Kern der Verhärtung im kleinen Gehirn bildete. Letztere ist wohl als Product eines, wahrscheinlich syphilitischen, Entzündungsprozesses aufzufassen, wenngleich eigentlich gummöse oder käsige Massen nicht vorgefunden wurden (cf. Virchow's Vorlesungen üb, Pathol, III. S.454 u. ff.).

Die vorstehend mitgetheilten Resultate der milsuslsopischen Untersuchung weisen darauf hin, dass in der Corticalsubstanz der Windungen des grossen Gehirns ein ähnlicher Prozess obgewaltet bat, wie er an der Dura mater als bämorrhagische Pachymeningitis schon fürdie Betrachtung mit blossem Auge deutlich genug vorlag. Hier wie dort finden wir die Residuen multipler capillärer Extravasationen als Zeichen wiederholter congestiver Attaquen. Bekanntlich bat man den die dũnnen Pseudomembranen der Dura mater bildenden Vorgang erst seit den Untersucbungen von Virchow und Hoffmann als einen, seinem inneren Wesen nach entzündlichen Vorgang, als bämorrhagische Pachymeningitis richtig gewürdigt, während man früher die Extravasation als das Wesentliche und Primäre anzusehen sich gewölnt batte. Wir sind nun aber um so mehr berechtigt ein analoges Verhältniss zwischen entzündlicher Reizung und Capillar-Extravasation auch für den Prozess in der Hirnrinde anzunebmen, als bier die Erscheinungen cerebraler Irritation (innere Beanruhigung, Schlaflosigkeit, zornige Affecte, Grössenwahn, ruheloses Umherwandern u. s. w.) den Lähmungs-Erscheinungen, namentlich a ch den paralytischen Anfällen vorhergingen und eine intensive Gemütlusdepression mit längerer irritirender Nachwirkung die nächste Veranlassung zum Ausbruche der Geistesstörung darstellte. Der habituelle Cerebralturgor spiegelte sich übrigens auch während der ersten Perioden des hiesigen Aufenthaltes in der andauernden Turgescenz des Gesichtes wieder.

Durch die fast vollständige doppelseitige Unterbrechung der Willisschen Communication, in Folge deren die Ausgleichung zwischen den arteriellen Stromgebieten der beiden Vertebrales einer - und der beiden Carotides internae andererseits für den Fall einer relativ vermehrten oder verminderten Blutzufubr in dem einen oder dem anderen Gebiete erschwert werden musste, mag die Entstehung localer Wallungen und capillärer Extravasationen begünstigt worden sein. Die Obliteration der Arteriae comm. selhst erscheint zum Theil wieder bedingt durch die Exostose auf dem Clivus, insofern durch das Hineinagen derselhen grade in die Bifurcation unter gleichzeitiger Mitwirkung der ungewöbnlich festen Adbäsion der den Circulus W. zusammensetzenden Gefässe an der Hirnoberfläche eine ganz bedeutende ond augenfällige Spannung der beiden genaníten Arterien bedingt, und ausserdem in Folge der mechanischen Insultation eine Verdickung der Wandungen und damit zugleich eine Verengung des Lumens der Art. basilaris herbeigeführt worden war.

\section{Die 4lste Versammlnng \\ dep Dentschen Naturforseler und Aerzte,}

zu der von uns auf die Tage rom 17 . bis 22 . September eingeladen worden war, wird, in Anbetracht der politischen Verhältnisse, dieses Jahr nicht stattfinden.

Frankfurt am Main, in Juni 1866 .

Herm. v. Heyer, erster Geschäftsführer.
Dr. med. Spiess sen., zweiter Geschäftsführer. 